\title{
Evaluation of a Multiple Integral of Tefera via Properties of the Exponential Distribution
}

\author{
Yaming $\mathrm{Yu}$ \\ Department of Statistics \\ University of California \\ Irvine 92697, USA \\ yamingy@uci.edu
}

Submitted: Jul 12, 2008; Accepted: Jul 21, 2008; Published: Jul 28, 2008

Mathematics Subject Classification: 26B12, 05A19, 60E05

\begin{abstract}
An interesting integral originally obtained by Tefera ("A multiple integral evaluation inspired by the multi-WZ method," Electron. J. Combin., 1999, \#N2) via the WZ method is proved using calculus and basic probability. General recursions for a class of such integrals are derived and associated combinatorial identities are mentioned.
\end{abstract}

\section{Background}

The integral in question reads

$$
\int_{[0, \infty)^{k}}\left(e_{2}(\mathbf{x})\right)^{m}\left(e_{1}(\mathbf{x})\right)^{n} e^{-e_{1}(\mathbf{x})} d \mathbf{x}=\frac{m !(2 m+n+k-1) !(k / 2)_{m}}{(2 m+k-1) !}\left(\frac{2(k-1)}{k}\right)^{m} T_{k}(m),
$$

where $k$ is a positive integer, $m$ and $n$ are nonnegative integers, $\mathbf{x}=\left(x_{1}, \ldots, x_{k}\right), e_{1}(\mathbf{x})=$ $\sum_{i=1}^{k} x_{i}, e_{2}(\mathbf{x})=\sum_{1 \leq i<j \leq k} x_{i} x_{j},(y)_{m}=\prod_{i=0}^{m-1}(y+i)$, and $T_{k}(m)$ is defined recursively by

$$
T_{k}(m)-T_{k}(m-1)=\frac{(k(k-2))^{m}((k-1) / 2)_{m}}{(k-1)^{2 m}(k / 2)_{m}} T_{k-1}(m), \quad m \geq 1, k \geq 2,
$$

and

$$
\begin{array}{cl}
T_{1}(m) & =0, \quad m \geq 0, \\
T_{k}(0) & =1, \quad k \geq 2 .
\end{array}
$$

Note that we are using an uncommon convention $0^{0}=0$ for the case $m=n=0, k=1$.

In [1], Tefera gave a computer-aided evaluation of (1), demonstrating the power of the WZ [2] method. It was also mentioned that a non-WZ proof would be desirable, especially if it is short. This note aims to provide such a proof. 


\section{A short proof}

This is done in two steps - the first does away with the integer $n$ using properties of the exponential distribution, while the second builds a recursion using integration by parts. In this section we denote the left hand side of $(1)$ by $I(n, m, k)$.

Proposition 1. For $n \geq 1$ we have $I(n, m, k)=(2 m+n+k-1) I(n-1, m, k)$.

Proof. Let $Z_{1}, \ldots, Z_{k}$ be independent random variables each having a standard exponential distribution, i.e., the common probability density is $p(z)=e^{-z}, z>0$. Denoting $\mathbf{Z}=\left(Z_{1}, \ldots, Z_{k}\right)$ we have

$$
\begin{aligned}
I(n, m, k) & =E\left(e_{2}(\mathbf{Z})\right)^{m}\left(e_{1}(\mathbf{Z})\right)^{n} \\
& =E\left(e_{1}(\mathbf{Z})\right)^{2 m+n}\left(\frac{e_{2}(\mathbf{Z})}{\left(e_{1}(\mathbf{Z})\right)^{2}}\right)^{m} \\
& =E\left(e_{1}(\mathbf{Z})\right)^{2 m+n} E\left(\frac{e_{2}(\mathbf{Z})}{\left(e_{1}(\mathbf{Z})\right)^{2}}\right)^{m} \\
& =\frac{(2 m+n+k-1) !}{(k-1) !} E\left(\frac{e_{2}(\mathbf{Z})}{\left(e_{1}(\mathbf{Z})\right)^{2}}\right)^{m}
\end{aligned}
$$

where we have used two properties of the exponential distribution: (i) $e_{1}(\mathbf{Z})$ is independent of $\left(Z_{1}, \ldots, Z_{k}\right) / e_{1}(\mathbf{Z})$ and hence independent of $e_{2}(\mathbf{Z}) /\left(e_{1}(\mathbf{Z})\right)^{2}$, and (ii) $e_{1}(\mathbf{Z})$ has a gamma distribution $\operatorname{Gam}(k, 1)$ whose $j$ th moment is $(j+k-1) ! /(k-1)$ !. The claim readily follows.

Proposition 2. For $k \geq 2$ and $m \geq 1$ we have

$$
I(0, m, k)=I(0, m, k-1)+\frac{m(k-1)(k+2(m-1))}{k} I(0, m-1, k) .
$$

Proof. Denote $\mathbf{x}_{-1}=\left(x_{2}, \ldots, x_{k}\right)$. Using integration by parts and exploiting the symmetry we obtain

$$
\begin{aligned}
I(0, m, k) & =\iint\left(e_{2}(\mathbf{x})\right)^{m} e^{-e_{1}(\mathbf{x})} d x_{1} d \mathbf{x}_{-1} \\
& =\int-\left.e^{-e_{1}(\mathbf{x})}\left(e_{2}(\mathbf{x})\right)^{m}\right|_{x_{1}=0} ^{\infty} d \mathbf{x}_{-1}+\iint m e^{-e_{1}(\mathbf{x})}\left(e_{2}(\mathbf{x})\right)^{m-1} e_{1}\left(\mathbf{x}_{-1}\right) d x_{1} d \mathbf{x}_{-1} \\
& =\int e^{-e_{1}\left(\mathbf{x}_{-1}\right)}\left(e_{2}\left(\mathbf{x}_{-1}\right)\right)^{m} d \mathbf{x}_{-1}+\frac{m(k-1)}{k} \int e^{-e_{1}(\mathbf{x})}\left(e_{2}(\mathbf{x})\right)^{m-1} e_{1}(\mathbf{x}) d \mathbf{x} \\
& =I(0, m, k-1)+\frac{m(k-1)}{k} I(1, m-1, k)
\end{aligned}
$$

where the limits of integration are omitted to save space. The claim now follows by Proposition 1.

To finish the proof of (1), we note that (i) by Proposition 1 it suffices to prove (1) for $n=0$, (ii) if we denote the right hand side of (1) by $J(n, m, k)$, then based on (2), after simple algebra $J(0, m, k)$ satisfies the recursion (3) as $I(0, m, k)$ does, and (iii) the boundary values of $I(0, m, k)$ and $J(0, m, k)$ match, i.e., $I(0, m, 1)=J(0, m, 1)=0$ for $m \geq 0$ and $I(0,0, k)=J(0,0, k)=1$ for $k \geq 2$. Thus $I(n, m, k) \equiv J(n, m, k)$. 


\section{General recursions}

This argument applies to a general class of integrals involving elementary symmetric functions. Specifically, let $e_{j}(\mathbf{x})=\sum_{1 \leq i_{1}<\ldots<i_{j} \leq k} x_{i_{1}} \ldots x_{i_{j}}, j=1, \ldots, k$, and consider the integral

$$
I_{k}\left(n_{1}, \ldots, n_{k}\right)=\int_{[0, \infty)^{k}} e^{-e_{1}(\mathbf{x})} \prod_{j=1}^{k}\left(e_{j}(\mathbf{x})\right)^{n_{j}} d \mathbf{x}
$$

for $n_{j} \geq 0,1 \leq j \leq k, k \geq 1$. Relation (1) corresponds to $n_{1}=n, n_{2}=m$ and $n_{3}=\ldots=n_{k}=0$. The following recursions are obtained by trivial modifications in the proofs of Propositions 1 and 2 .

Proposition 3. For $n_{1} \geq 1$ we have

$$
I_{k}\left(n_{1}, n_{2}, \ldots, n_{k}\right)=\left(k-1+\sum_{j=1}^{k} j n_{j}\right) I_{k}\left(n_{1}-1, n_{2}, \ldots, n_{k}\right) .
$$

Proposition 4. For $k \geq 2$ we have

$$
\begin{aligned}
I_{k}\left(0, n_{2}, \ldots, n_{k}\right)= & \delta_{k} I_{k-1}\left(0, n_{2}, \ldots, n_{k-1}\right) \\
& +n_{2} \frac{k-1}{k}\left(k+2\left(n_{2}-1\right)+\sum_{j=3}^{k} j n_{j}\right) I_{k}\left(0, n_{2}-1, n_{3}, \ldots, n_{k}\right) \\
& +\sum_{j=3}^{k} n_{j} \frac{k-j+1}{k} I_{k}\left(0, \ldots, n_{j-1}+1, n_{j}-1, n_{j+1}, \ldots, n_{k}\right)
\end{aligned}
$$

where $\delta_{k}=1$ if $n_{k}=0$ and $\delta_{k}=0$ if $n_{k}>0$.

Note that $I_{k}\left(n_{1}, \ldots, n_{k}\right)$ is given an arbitrary value if some $n_{j}<0$; this does not affect the recursion in Proposition 4.

Together with the boundary condition $I_{k}(0, \ldots, 0)=1, k \geq 1$, Propositions 3 and 4 determine $I_{k}\left(n_{1}, \ldots, n_{k}\right)$ for all $k \geq 1$ and $n_{j} \geq 0,1 \leq j \leq k$. It is doubtful whether these recursions are solvable in a simpler form. At any rate, we may calculate $I_{k}\left(0, n_{2}, \ldots, n_{k}\right), k \geq 2$, by building up a table of $I_{l}\left(0, m_{2}, \ldots, m_{l}\right)$ for values of $l$ and $m_{i}$ 's that satisfy $l \leq k, \sum_{j=2}^{l} m_{j} \leq \sum_{j=2}^{k} n_{j}$, and $m_{k} \leq n_{k}$ if $l=k$; this range can be further restricted if the largest $j$ for which $n_{j} \neq 0$ is less than $k$. We omit the details but include some values of $I_{3}\left(0, n_{2}, n_{3}\right)$ calculated this way in Table 1 .

It is reassuring to see that Table 1 contains only integer entries. This is not obvious from Proposition 4 but is so from (4), after expanding the product $\prod_{j=1}^{k}\left(e_{j}(\mathbf{x})\right)^{n_{j}}$ inside the integral. Alternatively, $I_{k}\left(n_{1}, \ldots, n_{k}\right)$ is a sum of products of various moments of the standard exponential distribution, and these moments are all integers. 
Table 1: Values of $I_{3}\left(0, n_{2}, n_{3}\right)$ for $n_{2}+n_{3} \leq 4$.

\begin{tabular}{c|rrrrr}
\hline$n_{2} \backslash n_{3}$ & 0 & 1 & 2 & 3 & 4 \\
\hline 0 & 1 & 1 & 8 & 216 & 13824 \\
1 & 3 & 12 & 216 & 10368 & \\
2 & 24 & 252 & 8640 & & \\
3 & 372 & 8208 & & & \\
4 & 9504 & & & & \\
\hline
\end{tabular}

\section{Associated combinatorial identities}

It would be interesting to know whether there exists a direct combinatorial interpretation of $I_{k}\left(n_{1}, \ldots, n_{k}\right)$ as defined by (4). In this direction we mention two associated binomial sum identities.

Let $Z_{1}, Z_{2}, \ldots$, be independent standard exponential random variables. For $n, m \geq 0$ we have

$$
\begin{aligned}
I_{2}(n, m) & =E\left(Z_{1}+Z_{2}\right)^{n}\left(Z_{1} Z_{2}\right)^{m} \\
& =\sum_{k=0}^{n} E\left(\begin{array}{l}
n \\
k
\end{array}\right) Z_{1}^{k+m} Z_{2}^{n-k+m} \\
& =\sum_{k=0}^{n}\left(\begin{array}{l}
n \\
k
\end{array}\right)(k+m) !(n-k+m) ! .
\end{aligned}
$$

On the other hand, (1) gives

$$
I_{2}(n, m)=\frac{(2 m+n+1) !}{(2 m+1) !}(m !)^{2} .
$$

Thus we obtain a familiar looking identity

$$
\left(\begin{array}{c}
2 m+n+1 \\
n
\end{array}\right)=\sum_{k=0}^{n}\left(\begin{array}{c}
k+m \\
m
\end{array}\right)\left(\begin{array}{c}
n-k+m \\
m
\end{array}\right), \quad m, n \geq 0 .
$$

Another instance of (1) is

$$
I_{3}(0, m, 0)=\frac{(2 m+1) !}{3^{m}} \sum_{k=0}^{m} \frac{3^{k}(k !)^{2}}{(2 k+1) !}, \quad m \geq 0 .
$$


We also have

$$
\begin{aligned}
I_{3}(0, m, 0) & =E\left(Z_{1} Z_{2}+Z_{1} Z_{3}+Z_{2} Z_{3}\right)^{m} \\
& =\sum_{0 \leq i, 0 \leq j, i+j \leq m} E \frac{m !}{i ! j !(m-i-j) !}\left(Z_{1} Z_{2}\right)^{i}\left(Z_{1} Z_{3}\right)^{j}\left(Z_{2} Z_{3}\right)^{m-i-j} \\
& =\sum_{0 \leq i, 0 \leq j, i+j \leq m} \frac{m !(i+j) !(m-j) !(m-i) !}{i ! j !(m-i-j) !}
\end{aligned}
$$

and after rewriting we get a less familiar but interesting identity

$$
\frac{(2 m+1) !}{3^{m}(m !)^{2}} \sum_{k=0}^{m} \frac{3^{k}(k !)^{2}}{(2 k+1) !}=\sum_{0 \leq i, 0 \leq j, i+j \leq m}\left(\begin{array}{c}
m-j \\
i
\end{array}\right)\left(\begin{array}{c}
m-i \\
j
\end{array}\right)\left(\begin{array}{c}
m \\
i+j
\end{array}\right)^{-1}, \quad m \geq 0 .
$$

Of course, (5) and (6) can be derived via alternative methods, for example the WZ method; the purpose of presenting them is mainly to draw attention to the potential of $I_{k}\left(n_{1}, \ldots, n_{k}\right)$ as combinatorial entities.

\section{References}

[1] A. Tefera, A multiple integral evaluation inspired by the multi-WZ method, Electron. J. Combin. 6 (1999), \#N2.

[2] H.S. Wilf and D. Zeilberger, An algorithmic proof theory for hypergeometric (ordinary and "q") multisum/integral identities, Invent. Math. 108 (1992), 575-633. 